\title{
Proposal of a System Architecture based on Emerging Technologies to motivate and engage STEM undergraduate students
}

\author{
E. López Zapata, Undergraduate Student ${ }^{1}$, E. Cajaleón Flores, Undergraduate Student ${ }^{1}$ \\ ${ }^{1}$ Universidad Nacional de Ingeniería, Perú, elopezz@uni.pe,ecajaleonf@uni.pe
}

\begin{abstract}
In this document, we propose a system architecture that aims to improve the performance and learning of basic sciences in STEM undergraduate students at Peruvian national universities. For this purpose, we first analyze the problems that arise in the most representative STEM university of Peru, the National University of Engineering (UNI by its initials in Spanish). Then, we review some papers with topics on STEM education, Human-Agent Interaction and Artificial Intelligence to finally propose the mentioned system and explain how it works. So, since the present document contains the first progress of a project that is currently under development, it will serve as a basis for future experiments, which will be defined and discussed at the end of this paper.
\end{abstract}

Keywords-- Engineering Education, Gamification, Human Agent Interaction, Intrinsic Motivation, Social Robotics.

\section{INTRODUCTION}

With the university reform that has currently been implemented in the Peruvian state, the seriousness of the problems of academic deficiency, which are presented in Peruvian national universities, has become evident. As an example, in the National University of San Marcos there were around 28,000 students who failed a course between one to four consecutive times, while in the National University of Engineering, there were at least over 2,000 students who failed a course 2 or more consecutive times.

In research literature, there are several works that indicate not only that STEM careers are the most difficult to manage, but also the first year is the most critical period in this type of careers $[1,2,3]$.

Additionally, [4] indicates that traditional didactic education is centered on the instructor, which means that the student bases his education on extrinsic motivation. References [3,4,5,11] suggest that in order to improve the learning of STEM courses, we should aim to develop an intrinsic motivation in students.

Different implementations of solution have been carried out to improve intrinsic motivation. A common thought in these implementations is the use of gamification as a pillar of the system $[4,6]$.

The terminology Gamification is usually used when we employ game components in non-gaming environments [7]. An example of a gamified system would be the used by shopping centers, which offer points and prizes when certain purchase conditions are met. Here we can identify game components such as challenges and rewards despite not being in a game environment.
From this review, we could infer that the solutions proposed in previous studies seek for the students to achieve their learning objectives on a self-motivated way, while he or she is trying to meet a challenge and receive rewards.

The mentioned solutions will be described in depth in the section Review of implemented solutions. However, we first need to demarcate our study scope and environment to come up with a solution. Therefore, we decided to investigate what are the conditions of the UNI and what are the bias values that characterize its students.

\section{PRELIMINARY STUDIES}

A. Current conditions of the National University of Engineering

A compilation of data on the student population was made. All of this data was obtained from the Strategic Plan of the UNI [8], from the statistics of OCAD-UNI (Central Office of Admission-UNI by its initials in Spanish) and from the registers of ORCE-UNI (Office of central registration and statistics-UNI by its initials in Spanish).

We managed to collect three different sets of data. The first set consists of the number of UNI entrants from the period 2005 to $2014(\mathrm{M}=1624.2, \mathrm{SD}=181.437)$. With this data, a linear regression was performed with $\mathrm{R}^{2}=0.944$ to predict the values until the year 2021.

The second set consists of students enrolled in the university from 2005 to 2018 ( $\mathrm{M}=10921.64, \mathrm{SD}=494.27)$. With this second data set, a polynomial regression of order 2 was performed with $\mathrm{R}^{2}=0.96$.

The third set of data is made up of students who receive their bachelor degree. Since we only have data from 2005 to $2012(\mathrm{M}=1213.75, \mathrm{SD}=163.5)$, in order to complete an accurate prediction of the values until the year 2021, we performed a logarithmic regression with $\mathrm{R}^{2}=0.807$. It should be noted that the linear regression had an $\mathrm{R}^{2}=0.68$.

The three data sets, with the predicted data set, are presented in Fig1. It is observed that there is a growing trend both in the number of entrant students and in the number of bachelor students.

This does not exhibit any strange behavior but shows that the university is in constant growth, and is able to offer a larger number of vacancies for new students.

However, there is a trend of growth in the number of students enrolled in the university. This shows us a future problem since our model predicts that in the year 2021 there 
will be a population of approximately 13,000 students, which means an increase of 1000 students in only 3 years.

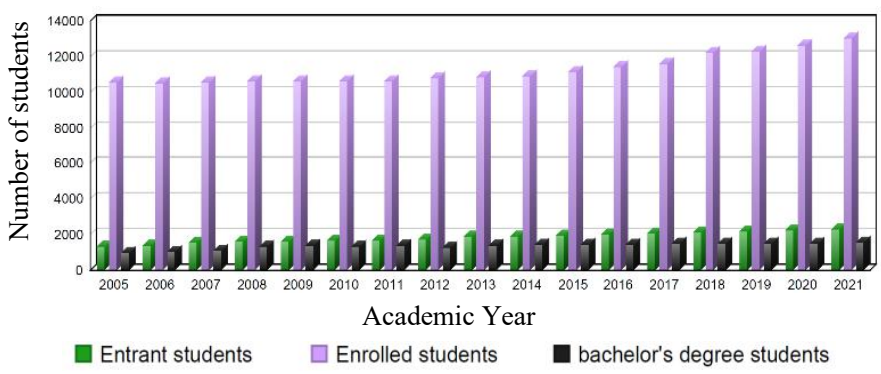

Fig. 1 Students distribution at National University of Engineering.

We infer that there are two sources that lead to the increase of enrolled students in the university. The first is a regular source, product of the difference between the amount of students that are currently entering the university compared to those who did it 5 years ago. Due to the gap caused by the 5 years of studies, it is natural that the number of admitted students is greater than the number of graduates.

However, if only this source of student accumulation existed, the increase in enrolled students would be linear. Since growth has a polynomial grade 2 trend, we can infer that there is another source of student accumulation. This second source is the product of the students that stayed at the university because they could not pass all their needed courses to complete their major.

To reinforce this idea, the scores obtained by the students of the National University of Engineering are shown in Fig. 2. These data were obtained from the Strategic Plan of the UNI $[8]$.

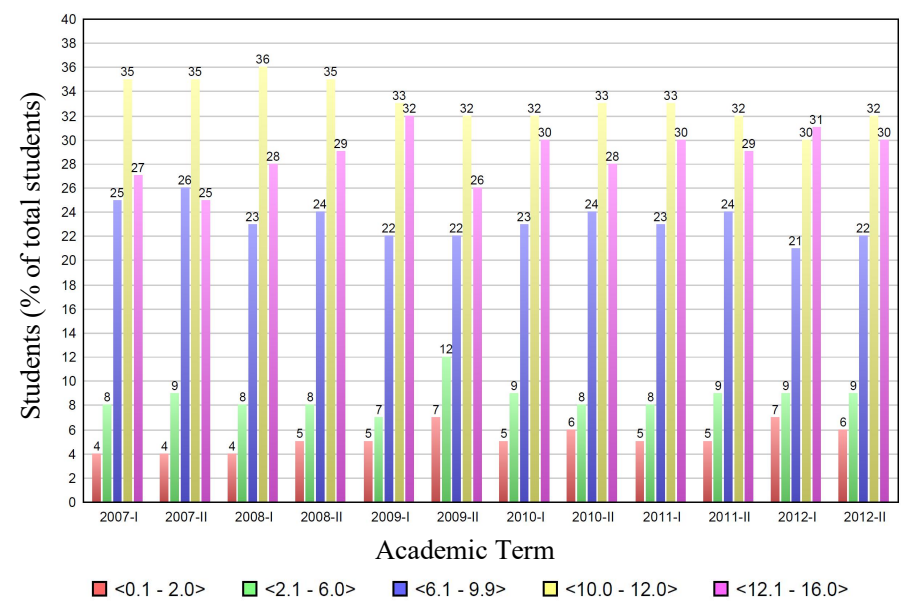

Fig. 2 Student scores distribution at National University of Engineering.

The UNI scores are on a scale of 0-20, where a grade of 10.0 means that the student was able to pass the course. According to Fig. 2, the average grade in the different academic terms is in the range of $<9.54-10.27>$. That means total students average score in some terms is a fail grade.
In addition, if we analyze the percentage score distribution by term, we will find that a mean of $37 \%$ of the total number of students failed in their courses $(\mathrm{SD}=1.8)$.

The data shown allows us to clarify that there is a problem in the UNI. We need to find out what STEM students perceive about the problem.

For this, a survey was conducted among the students of the School of Mechanical Engineering of the UNI. We had the participation of $7.8 \%$ of the total population (around 130 students).

In the last question of this survey a free response was allowed. We asked students about what do they consider should be improved in the university to achieve a better academic performance.

As expected, there was a variety of responses. However, a tendency was observed within them. Most students consider that the student's performance is supported by the work of both the teacher and the student him or herself. Therefore, some of them emphasize that either teachers should be trained in teaching methodologies or change them, while others consider that the student must change his attitude and focus on improving his or her performance.

\section{B. Review of implemented solutions}

There are different works which have implemented solutions to motivate and engage STEM undergraduate students. Within the literature reviewed, a first group of works implemented pre-professional practices simulators.

One example of this type of works is [6], which evaluated the benefits of using an educational game in the first year of undergraduate degree programs in industrial engineering. Its system is quite simple since it is based on a predefined model, which current output is a function of the previous values of its inputs, which are decided by the students. Its implementation is based on a web environment. There, students send data through the game platform (business administration simulator) and a central computer calculates the respective performance of the decisions made. The importance of [6] is that its results add empirical evidence that learning through educational games based in decision making is a viable method since it increased the student's intrinsic motivation and their interest in topics related to their career.

References $[1,10]$ apply a system similar to the one mentioned since they are also based on decision making. However, each of these works implements the system in a different way. Ref. [10] is based on a simulator that shows a high school student the result of his or her structural design. This work concentrates its efforts in verifying the effectiveness of the use of this type of systems in the teaching of Physics subjects, due to the fact that in 2007 there were still not many researches about it.

Ref. [1] applies a web-based pre-professional practice simulator, but unlike [6], it does not have a model that calculates the performance of decisions made by the student. Instead, this work implements a messaging center through which you can send reports that summarize your decision

$1^{\text {th }}$ LACCEI International Multi-Conference for Engineering, Education, and Technology: "Innovation in Education and 
making and design selection. Therefore, it does not restrict the decision options that the student may take. However, this requires mentors who are constantly reviewing the reports presented by the different student teams.

The references $[9,11]$ follow the same line of methodology, proposing a system that allows students to develop an intrinsic motivation and bring it closer to the processes carried out in their career. However, the implementation of their systems differs from the works presented previously.

Ref. [9] implements an internship in a laboratory, where the first-year undergraduate students are assigned a research project, so that they are related to immediate applications to their career. This proposal is based on the pillars established by [3], which are: Early research, Active learning and learning communities. These pillars are necessary for the "identify as a scientist" objective, which allows increasing the motivation, confidence, and persistence of students in STEM careers [3].

On the other hand, [11] proposed a system of education based on gamification and built in Virtual Reality. Emphasis is placed on the fact that this system works particularly well in science, engineering and medicine careers, since this type of careers requires active learning, which is more related to kinesthetic learning.

\section{PROPOSED SYSTEM ARCHITECTURE}

\section{A. Design guidelines for the proposed system}

Ref. [1] not only implemented a pre-professional practice simulator but also proposed that this system should consider some critical aspects of engineering design. We consider 5 of these aspects: individual research, feedback, teamwork, design selection, and presentation of results. From the survey conducted among 131 students of the School of Mechanical Engineering of the UNI, the importance that the students perceive about these 5 aspects was obtained. The results of the survey are shown in Fig. 3.

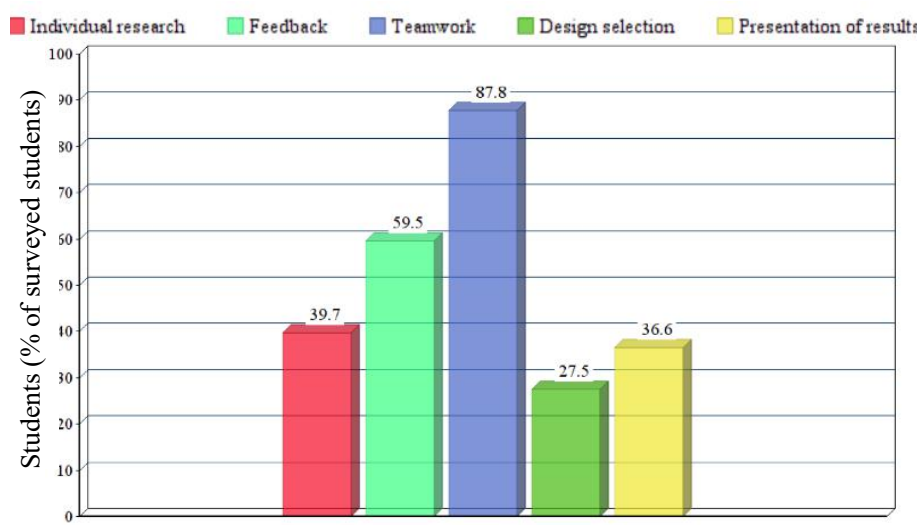

Fig. 3 Perceived importance of the most 5 important aspects of a engineering project.

The values shown in Fig. 3 suggest that STEM students at national universities value teamwork above all other aspects to be considered in a project. To be specific, the $87.8 \%$ of surveyed students think Teamwork is important when they're working on a project. This means that the system we propose should focus on improving Teamwork, without neglecting the other 4 important aspects of engineering design.

In the same survey we also asked about the importance of the pillars which are established in [3]. As we mentioned in the previous section, there are 3 pillars: Early research, Active learning and learning communities. In Fig. 4 we can observe a bar graph which summarizes the students' answers.

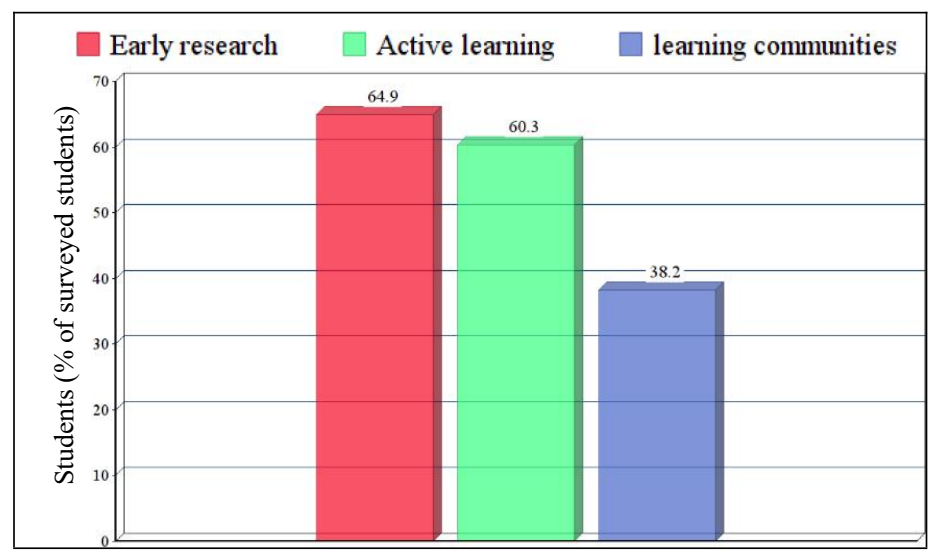

Fig. 4 Perceived importance of the 3 pillars necessary for the "identify as a scientist" objective.

It is observed that students consider early research and active learning more important. This means that if we succeed in improving these two aspects of the student, he or she will achieve the motivation and confidence of their own abilities to achieve the objectives proposed in their career (as a result of getting identified as a scientist).

On the other hand, there is an additional feature that none of the previous work has considered in the design of its system. This feature is the nature of time. In the works presented in the previous sections, the implementation of the systems was based on a synchronous access to the system. That means there was a predefined schedule for researchers to control access to the system.

Nevertheless, the system we propose in the present work should be based in an asynchronous access. The reason arises after conducting a different survey to the students of the School of Mechanical Engineering. In this survey, we asked them about when do they feel more comfortable and relaxed. There were 4 different options for this question. The results are shown in Fig. 5.

As we can see, the period of the term in which students feel more relaxed and comfortable is in the period of practices.However, it must be admitted that the distribution of students in the other options is fairly uniform. That is why we accept that different students will have different periods of time which they can take advantage of to complement their studies.

16 $^{\text {th }}$ LACCEI International Multi-Conference for Engineering, Education, and Technology: "Innovation in Education and Inclusion", 19-21 July 2018, Lima, Peru. 


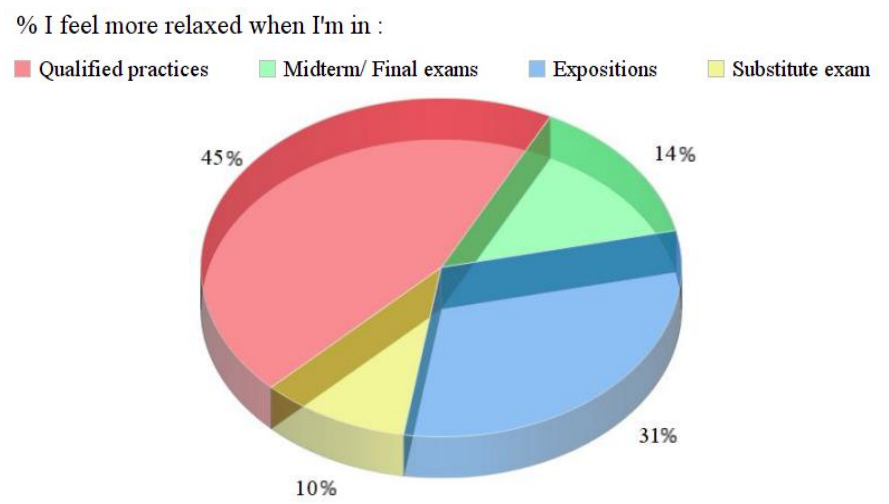

Fig. 5 Periods of the term when students feel more comfortable and relaxed.

\section{B. Design process for the proposed system}

The proposed system consists of a game that will run as an application on a smart-phone. This decision is supported by [1], which states that the system must be designed so that its implementation does not require a large investment. It should be noted that is only feasible since the majority of STEM students own a smart-phone.

In the next lines, the proposed system will be explained at different levels.

1)Information System Architecture: As already mentioned, a mobile application will be used as the user interface. This application will be programmed in a way that allows us to collect data from the student. The information flow will begin with the actions that the student makes within the platform, and will continue with the sending of these as codified information from the application to a database. Remotely, a computer will run the analysis and prediction algorithms to choose the optimal interaction responses that the mobile application should perform based on the database collected. Fig. 6 shows a block diagram describing the operation explained.

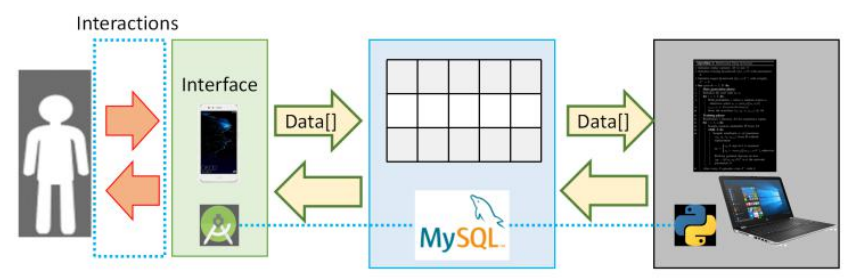

Fig. 6 Block diagram describing the information flow.

After observing the flow of information, it should be clarified what type of information is expected to receive and what kind of interactions the application will perform. For this, we review works belonging to the field of HRI (Human Robot Interaction), such as $[12,13,14]$. Within the methodology of [12], it is highlighted the prediction of emotions through the use of the Affdex mobile SDK (Affectiva, Inc., http://affectiva.com). This platform will allow us to obtain the mood of the user without the need to increase the computational load in the central computer.

In Ref. [14] it can be observed that the inertial sensors inside a small robot provide data that are not only useful for dynamic studies, but also allow us to classify the moods of the human that interact with the small robot. This gives a first approach to the idea that the data sensed by the gyroscope of a smartphone can be used to predict the user's moods.

The analysis carried out in [16] reinforces the previous idea. In this work, the statistical relationships between the data provided by the sensors located in a smartphone and the behavioral classifiers belonging to their owners are shown. It should be noted that this study was conducted with a population made up of undergraduate students. The results of this work are very important because after having found a correlational relation among our data of interest, we can state that the application of artificial intelligence on this model will not be in vain. For this reason, as in [14] a machine learning algorithm (SVM) was applied, we can also apply classification algorithms in our system, among which SVM is included.

2)Stages of achievement: In order to complement the idea of the proposed system, it is necessary to obtain a general process diagram that contains the stages that the user will experience. For didactic reasons, a flowchart is used, which can be seen in Fig 7.

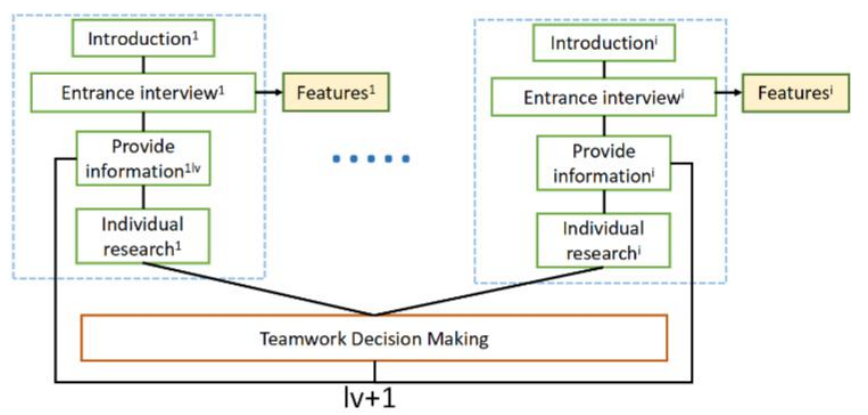

Fig. 7 Flowchart of the processes that the user (student) will experience.

An introductory process can be distinguished. Here, the context of the game will be explained to the student. So, this stage is aimed at capturing the user's attention. The challenges and rewards that await within the new system will be presented. After this, the student will be guided to an interview, so that we can obtain a set of user characteristics and we can add it to a database hosted in the cloud, such as MySQL.

After this, the system will make available to the user the first amount of information, which will be necessary to make a correct decision in the next stage. However, the information provided will only serve to guide the student in the topics that must be investigated to solve the challenge that is posed. The resolution of the challenges will allow progress in the game so that the student can achieve the goal of completing it. 
Because the guidelines specified that the system should be oriented to the development of teamwork, the following 2 stages are proposed in a way that will allow students to improve their teamwork skills.

In order to achieve this, the system is proposed in such a way that it is not possible to advance without making an appropriate decision. As shown in Fig. 7, the information provided to "i" people is required to make an accurate decision. This will lead to the formation of work groups formed by "i" people.

After achieving a correct decision making, the progress of the game will be updated and the progress of the game will be increased. In fig. 7, it is observed that a loop will be made increasing in 1 the value of a variable "lv".

The states characterized by a specific value of "lv" will be named "stages of achievement" and allow the groups to compete in a leader-board. If sponsorship is achieved, the winning team could receive a prize, so that the well-done work is encouraged.

3)Information management system: The flow of information shown in Fig. 6 makes it seem that the system works in a static way, like the works shown in $[6,10]$. However, the input data does not correspond to a fixed output value, but the model changes over time.

Fig. 8 shows a simplification of the model proposed for an instant " $\mathrm{t}$ ", with information provided "Ln" for a stage of achievement "lv" and a user with tag "tg". In the loop described by "lv", a group decision will be made, which will be graded with a variable "Os". We call this variable "objective score".

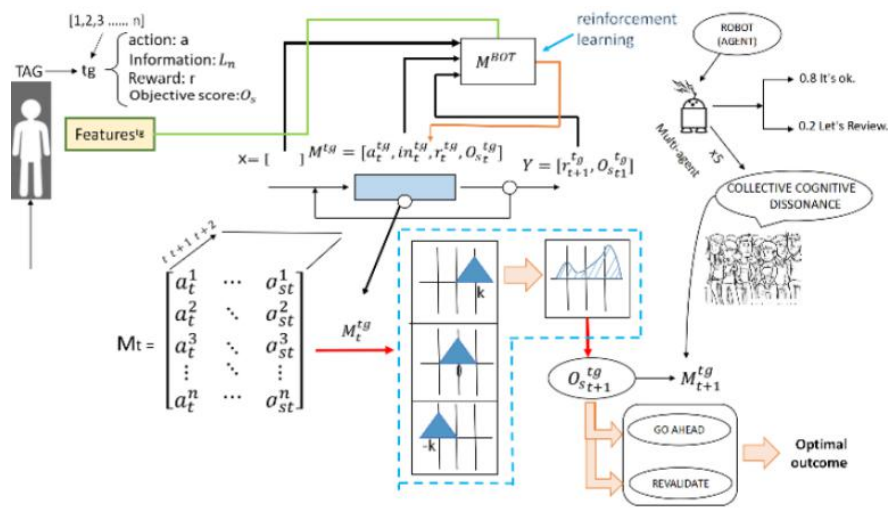

Fig. 8 Simplified model's operation description.

It is expected to have as input to the analysis and prediction model an action "a", a state of previous reward "r" and an objective score "Os". The output of the model will be a reward "r" and an objective score "Os". It should be noted that each considered variable belongs to a tag "tg".

In figure 8 the system is described as one based on fuzzy logic. This is not mandatory; therefore we note that the best artificial intelligence model for our proposed system must still be found.
On the other hand, at the moment of qualifying the decision made by the team through the value "Os", in the case that the group has made an incorrect decision, it is proposed to take advantage to feedback the group. This decision is based on the result of the survey shown in fig. 3. The feedback will be based either on improvement advice or specifications about what is required by the objective, according to predictions made by a second information model, which we will call BOT.

This second data model is proposed due to the study presented in [13]. Here it was studied how an agent affects the decision making and mentality of a student after a process of cognitive dissonance. A process of this kind occurs when a conflict of preconceived ideas and ideas contrary to it appears. In our case, the participants will be sure that their decision was the most appropriate, but our first model will affirm the opposite. To take advantage of this situation, our second model will perform a feedback.

In the study conducted in [13], the agent was a robot and it was shown to be more effective than a virtual system, however our proposal is to implement only the artificial intelligence logic that governs the agent. Future studies are planned to validate that a robotic agent provides sufficient benefits to justify an investment of implementation.

To achieve that our BOT model can interact with the users in the best possible way, it is proposed that reinforcement learning will be used. There are works like $[12,15]$ that certify the validity of this type of algorithms. Especially [15] achieved a robot that could exhibit social intelligence in its interactions with humans, after 14 days of adaptation.

As seen in fig. 8, the sensed data and the user's own characteristics (obtained thanks to the data sensed by a smartphone and a classification algorithm) will be used directly by our BOT model to be able to perform their actions in the most effective way possible. It is proposed that this model, in addition to giving feedback to the students, also encourages them to restructure their collective decision and to resubmit a new decision for revalidation, that could lead them to obtain a new of "Os".

It should be noted that in future work it is expected that this second model can perform additional interactions to motivate students, achieving extrinsic motivation in addition to the intrinsic motivation developed by the complete system.

\section{DISCUSSION}

The current proposal was based on implementations that have worked in their respective implementation environments. Nevertheless, it will be required to validate if the considered systems have the same effect on the STEM undergraduate students of the National University of Engineering. In addition, the synergy recommended in the present work must be validated. It is mainly necessary to study the behavior of IA-based agents when they manage a system that simulates an internship. It is expected to obtain good results due to the

$1^{\text {th }}$ LACCEI International Multi-Conference for Engineering, Education, and Technology: "Innovation in Education and 
success that this type of agents have had in other educational platforms.

On the other hand, it is noted that the system has only one way of interaction with the user, which is through the interface of the mobile application. This limitation was made based on the Peruvian reality, since implementing one robot per student would increase the implementation cost too much. However, it is recommended to evaluate the degree of benefit that a physical agent would bring before a virtual one, and how complex it should be. In this way, a cost-benefit relationship can be obtained, which could be evaluated according to the country of implementation.

Finally, the first study to be carried out as the next phase of the present project is presented. This first study is presented when we analyze the BOT agent. Leaving aside their means of interaction, there is a fundamental difference in the working environment between the BOT model and the agent implemented by [13]. This is because, in our system, the phenomenon of cognitive dissonance will be presented in each of the students that make up the teamwork. Therefore, the consonance-dissonance ratio will be different in each of them. In addition, each of these students may play the role of agent at the time of interaction. This means that there will be a collective cognitive dissonance, in a multi-agent system. The question that is beyond the state of the art and our present work is as follows: How does an external agent affect a multiagent system in which collective cognitive dissonance occurs? Does the result change when adding external agents?

\section{CONCLUSION}

Given the public statistics of OCAD-UNI and ORCE-UNI and the Strategic Plan of the UNI, we managed to describe the current situation of the students in the UNI. As we can see in the statistics given, there is a large number of students who remain more than 5 years. This situation, along with the low grades present at the same institution, allow us to conclude that there is a problem with STEM education in Peruvian national universities. Because this is not a problem unrelated to the global reality, we were able to find different works that have implemented systems to motivate and engage STEM undergraduate students. We realized that these works seek to motivate and engage students because this leads to improve the learning experience and therefore the quality of this learning. Even [9] exposes results that show that students' grades are significantly increased as a result of this better learning.

Moreover, a system based on the reviewed papers and the requirements of the students was proposed. This system makes use of two types of artificial intelligence: regression / classification models and reinforcement learning models. By working with these two models, an automatic management of information is achieved and interesting research topics are opened for the field of Human Agent Interaction. Therefore, in the discussion section, the foundations are laid for future work in the present project.

\section{REFERENCES}

[1] N.C.Chesler et al, "Design of a Professional Practice Simulator for Educating and Motivating First-Year Engineering Students", Advances in Engineering Education, American Society for Engineering Education, 2013.

[2] W.C. Lee, C.N.Seimetz, and C.T.Amelink, "Examining the Transition To Engineering: A Multi-Case Study of Six Diverse Summer Bridge Program Participants", 121st ASEE Annual Conference \& Exposition, American Society for Engineering Education, 2014.

[3] M.J.Graham, J.Frederick, A.Byars-Winston, and A.Hunter, "Increasing Persistence of College Students in STEM", SCIENCE, vol.341, pp. 14551456, 2013.

[4] J.Bafield, and B.Wilkerson, "Increasing Student Intrinsic Motivation And Self-Efficacy Through Gamification Pedadogy", Contemporary Issues In Education Research - Fourth Quarter 2014, vol. 7, no. 4, 2014.

[5] C.Mega, L.Ronconi, and R.De Beni, "What Makes a Good Student? How Emotions, Self-Regulated Learning and Motivation Contribute to Academic Achievement", Journal of Educational Psychology, vol. 106, no. 1, pp. 121.131, 2014.

[6] L.Falavigna, J.Duarte, A.Dittmar amd M.Pizzolato, "Benefits of educational game as an introductory activity in industrial engineering education", Computers in Human Behavior, vol. 58, pp. 315-324, 2016.

[7] S.Deterding, M.Sicart, L.Nacke, K.O'Hara, and D.Dixon, "Gamification. using game-design elements in non-gaming contexts", Proceedings of the 2011 annual conference extended abstracts on Human factors in computing systems , pp. 2425-2428, May, 2011.

[8] OCPLA-UNI, "Plan Estratégico Institucional UNI 2014-2021" unpublished.

[9] Tuajanda C. Jordan et al, "A Broadly Implementable Research Course in Phage Discovery and Genomics for First-Year Undergraduate Students", mBio, vol. 5, no. 1, January, 2014.

[10]G.Navoa, and D.Williamson, "SodaConstructing Knowledge through Exploratoids", Journal of Research in Science Teaching, vol. 44, no. 1, pp. 133-153, 2007.

[11]C.Fernández, G.Esteban, M.C Conde and F.J.Garcia, "Improving motivation in a haptic teaching/learning framework", International Journal of Engineering Education (IJEE), 32(1B), pp. 553-562, 2016.

[12]S.Jeong, and C.Breazeal, "Toward Robotic Companions that Enhance Psychological Wellbeing with Smarthphone", ACM/IEEE International Conference on Human-Robot Interaction, pp. 345-346, 2017.

[13]K.Youssef, J.Ham and M. Okada, "Investigating the Effects of the Persuasive Source's Social Agency Level and the Student's Profile to Overcome the Cognitive Dissonance", 8th International Conference on Social Robotics, pp. 115-125, 2016.

[14]M.D Coneey, S.Nishio and H.Ishiguro, "Designing Robots for Wellbeing: Theoretical Background and Visual Scenes of Affectionate Play with a Small Humanoid Robot", Lovotics, vol. 1, no. 1, 2014.

[15]A.Hussain, Y.Nakamura, Y.Yoshikawa and H.Ishiguro, "Robot gains Social Intelligence through Multimodal Deep Reinforcement Learning", IEEE-RAS International Conference on Humanoid Robots, 2016.

[16]R.Wang et al, "StudentLife: Assessing Mental Health, Academic Performance and Behavioral Trends of College Students using Smartphones", UbiComp'14, 2014.

16 $^{\text {th }}$ LACCEI International Multi-Conference for Engineering, Education, and Technology: "Innovation in Education and Inclusion", 19-21 July 2018, Lima, Peru. 